\title{
Observation of the Fresnel and Arago laws using the Mach-Zehnder interferometer
}

\author{
Bhaskar Kanseri, ${ }^{\text {a) }}$ Nandan S. Bisht, and H. C. Kandpal ${ }^{\text {b) }}$ \\ National Physical Laboratory, Dr. K. S. Krishnan Road, New Delhi 110012, India \\ Shyama Rath \\ Department of Physics and Astrophysics, University of Delhi, Delhi 110007, India
}

(Received 28 May 2007; accepted 13 September 2007)

\begin{abstract}
An experimental study is conducted to determine the effect of polarization on the interference of light waves. By using the temporal coherence property of light in a Mach-Zehnder interferometer, we verified the four important Fresnel and Arago laws for linearly polarized and circularly polarized light. This experiment provides a simple method for undergraduates to study the phenomena of interference and polarization. (C) 2008 American Association of Physics Teachers.
\end{abstract}

[DOI: $10.1119 / 1.2794349]$

\section{INTRODUCTION}

The interference of light is the strongest evidence for the wave theory of light. The superposition of light waves produces a fringe pattern due to interference. The visibility of the fringes is strongly governed by the state of polarization of the light, which is responsible for the production and extinction of fringes. More than 200 years ago, Fresnel and Arago ${ }^{1}$ gave four laws governing the superposition of light waves on the basis of the experiments they performed. The four laws can be stated as follows.

(1) Two rays of light polarized in the same plane interfere like rays of ordinary (natural/unpolarized) light, so that in both cases the phenomena of interference is identical (to ordinary light).

(2) Rays of light polarized at right angles do not produce any interference effects on each other under the same circumstances.

(3) Two rays that were originally polarized at right angles may be brought to the same plane of polarization without thereby acquiring the ability to interfere.

(4) Two rays of light polarized at right angles and then brought into the same plane of polarization interfere like ordinary light if they were originally polarized in the same plane.

Light waves were initially considered to be longitudinal waves and hence the results of the experiments performed by Fresnel and Arago were puzzling. An elementary explanation of the results was given by Young who treated the light vibrations as transverse. ${ }^{2}$ In 1852 Stokes $^{3}$ introduced four parameters (now known as the Stokes parameters) in his mathematical description of polarized light. In the last three decades the Fresnel and Arago laws were given a modern formulation using the Stokes parameters. ${ }^{4-}$ Recently these laws have been generalized for any state of coherence and polarization of the light. ${ }^{8,9}$

Mellen ${ }^{10}$ used a Michelson interferometer with quarter wave plates to demonstrate these laws. This method was somewhat difficult because a nonfunctioning quarter wave plate was used to compensate the two optical paths. To obtain sharp fringes, Fortin ${ }^{11}$ used a laser and Young's twobeam interferometer with a double-slit made of thin blade edges. Weak intensity interference fringes were obtained at the wall. A realization of this setup was difficult because the separation between the two slits was too small to introduce the polarizers in separate paths. The complexities of these experiments were overcome by Henry ${ }^{12}$ using a Ronchi grating as a beam splitter. The amount of light available in the fringe plane was much greater than for the previous methods, but the orientation of polarizers was still difficult. Ferguson ${ }^{13}$ used a birefringent calcite crystal with a converging lens to produce a bright interference fringe pattern. A highly polished, good optical-quality crystal is required.

We used a simple setup, a Mach-Zehnder interferometer, which works on the principle of temporal coherence. ${ }^{14}$ This interferometer is an extension of the Michelson interferometer and, due to $90^{\circ}$ separate beams, it is easy to insert polarizers and rotators in two separate paths of the light beam. The attractive feature of the Mach-Zehnder interferometer is that the two beams are widely separated and traverse the polarizers only once. An extended source can be used with interference fringes localized in any desired plane. This simple setup demonstrates the fundamental relation between polarization and interference and enables us to study the Fresnel and Arago laws in a most convenient way. ${ }^{15,16}$

\section{EXPERIMENTAL METHODS AND RESULTS}

An inexpensive $\mathrm{He}-\mathrm{Ne}$ laser (Newport model No. P-30988, $\lambda=632.8 \mathrm{~nm}, \quad$ power $=2 \mathrm{~mW}$, beam diameter $=0.8 \mathrm{~mm}$ ) with random polarization was used as a quasimonochromatic source. The laser beam with input beam diameter $=1 \mathrm{~mm}(\max )$ was passed through a (Newport) beam expander with an expansion ratio $10 \times$. The expanded beam was collimated using a crown glass convex lens of focal length $20 \mathrm{~cm}$, and a beam of approximately $2 \mathrm{~cm}$ diameter was obtained (see Fig. 1). This collimated beam was then passed through a broadband nonpolarizing 50:50 cube beam-splitter $\mathrm{BS}_{1}$ (Newport, wavelength range 400 to $700 \mathrm{~nm})$. The transmitted and the reflected beams at $\mathrm{BS}_{1}$ were mixed again at beam splitter $\mathrm{BS}_{2}$ (with the same specifications as $\mathrm{BS}_{1}$ ) after reflection from two broadband, frontcoated (Al) mirrors $\mathrm{M}_{1}$ and $\mathrm{M}_{2}$, respectively (see Fig. 1). Fringes were obtained on the planes of observation $\mathrm{R}$ and $\mathrm{R}^{\prime}$. The phase difference between the two fringe patterns was $180^{\circ}$. Therefore, any plane of observation $\left(\mathrm{R}\right.$ or $\left.\mathrm{R}^{\prime}\right)$ can be chosen for study. The fringes with maximum sharpness were obtained on a screen (plain white paper) placed in the plane $\mathrm{R} .{ }^{17}$ The fringes were photographed using a high-resolution 


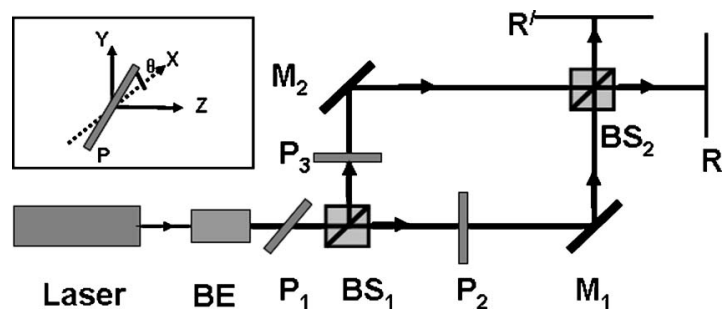

Fig. 1. Experimental arrangement of the Mach-Zehnder interferometer needed to verify the first and second Fresnel and Arago laws using linear polarization. $\mathrm{BE}$ stands for beam expander, $\mathrm{BS}_{1}$ and $\mathrm{BS}_{2}$ are beam-splitters, $\mathrm{P}_{1}, \mathrm{P}_{2}$, and $\mathrm{P}_{3}$ are polarizers, $\mathrm{M}_{1}$ and $\mathrm{M}_{2}$ are front-coated mirrors, and $\mathrm{R}$ and $\mathrm{R}^{\prime}$ are observation planes. (Inset) The $X, Y, Z$ axes are shown. The angle between the polarizer and the $X$-axis is shown as $\theta . Z$ is the direction of propagation of the beam.

digital camera. To minimize mechanical vibrations the experimental setup was built on a vibration isolation table. The Fresnel and Arago laws were verified as follows.

To verify the first law, a polarizer $\mathrm{P}_{1}$ (wavelength range 430 to $670 \mathrm{~nm}$ ) with direction of polarization $45^{\circ}$ with respect to the $X$-axis (shown as $\theta$, in the inset of Fig. 1) was placed before the first beam-splitter $\mathrm{BS}_{1}$ of the MachZehnder interferometer. The emerging beam was linearly polarized at $45^{\circ}$ with respect to the $X$-axis (Fig. 1) and its intensity reduced to half of the incident beam. Polarizers $\mathrm{P}_{2}$ and $\mathrm{P}_{3}$ (with the same specifications as $\mathrm{P}_{1}$ ) with direction of polarization along the $X$-axis were placed in the paths of the separate beams after the first beam-splitter $\mathrm{BS}_{1}$ (Fig. 1). The interference fringes obtained on the observation plane showed no change in sharpness [Fig. 2(a)]; however, some change in the intensity was recorded due to the absorption of light by the polarizers. Interference in the two beams was observed when both beams had the same direction of polarization. Thus the first law was verified.

Rotating the polarizer $\mathrm{P}_{1}$ made the fringes disappear because the planes of polarization of $\mathrm{P}_{1}$ and $\mathrm{P}_{2}$ or $\mathrm{P}_{3}$ were orthogonal. For intermediate positions of $\mathrm{P}_{1}$, the intensity of the fringes followed the Malus law. ${ }^{14}$ When the direction of polarization of $\mathrm{P}_{1}$ and $\mathrm{P}_{2}\left(\right.$ or $\left.\mathrm{P}_{3}\right)$ was the same, the fringes reappeared and the sharpness of fringes was a maximum [Fig. 2(b)].

If either of the polarizers $\mathrm{P}_{2}$ or $\mathrm{P}_{3}$ is rotated relative to the other (see Fig. 1), the fringes begin to disappear and, for $90^{\circ}$ cross-polarization (orthogonal polarization), the interference fringes vanished completely. This observation showed that light waves with orthogonal polarization do not interfere [Fig. 2(c)]. Because the noncorrelated orthogonal components of the light fields superpose, no fringes were seen on the plane of observation. Rotating the direction of polarization of $\mathrm{P}_{1}$ did not recover the fringes because all the directions of polarization of $\mathrm{P}_{1}$ were always mutually orthogonal to the directions of polarization produced by $\mathrm{P}_{2}$ or $\mathrm{P}_{3}$. This result verified the second law.

To verify the third law, polarizer $\mathrm{P}_{1}$ was removed and another polarizer $\mathrm{P}_{4}$ (identical to $\mathrm{P}_{1}$ ) was introduced just after the beam splitter $\mathrm{BS}_{2}$ (see Fig. 3). The beams in the two arms of the interferometer after $\mathrm{P}_{2}$ and $\mathrm{P}_{3}$ were orthogonally polarized. Fringes could not be produced on the observation plane for any direction of polarization of polarizer $\mathrm{P}_{4}$ [see Fig. 4(a)]. This observation verified the third law.

In this case the two orthogonally polarized components originated from different components of the electric field,

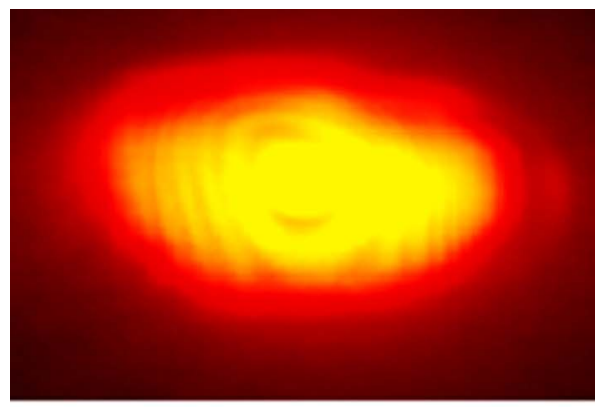

(a)

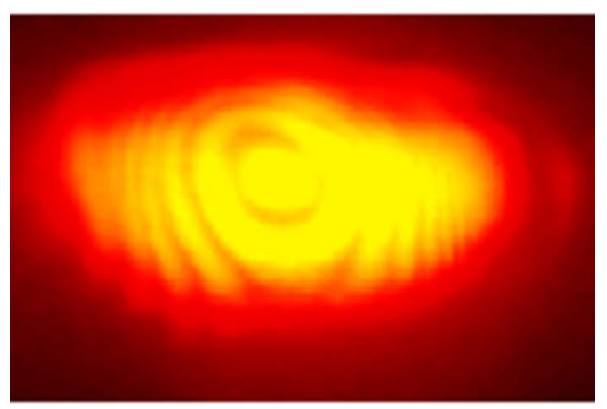

(b)

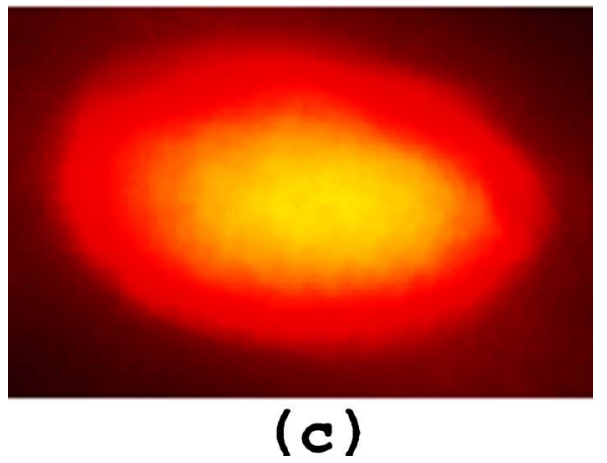

Fig. 2. (Color online) Interference fringes obtained for linearly polarized light verifying the first and second Fresnel and Arago laws. (a) Polarizers $\mathrm{P}_{2}$ and $\mathrm{P}_{3}$ produce light beams with the direction of polarization along the $X$-axis. (b) $\mathrm{P}_{2}$ and $\mathrm{P}_{3}$ produce light beams with the same direction of polarization as that produced by polarizer $\mathrm{P}_{1}$. (c) Polarizers $\mathrm{P}_{2}$ and $\mathrm{P}_{3}$ produce orthogonally polarized light beams.

interference did not occur, and hence no fringes were observed. Because the source was not strictly unpolarized (in practice light is always partially polarized), some correlation might have existed between the orthogonal components of the beam generated from the source. For these conditions the

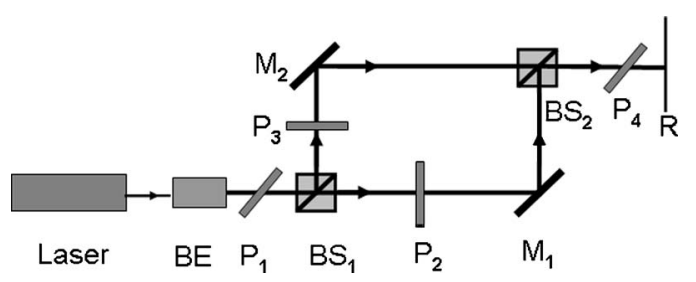

Fig. 3. Experimental arrangement for verifying the third and fourth Fresnel and Arago laws. $\mathrm{P}_{4}$ is a polarizer making a $45^{\circ}$ angle with the $X$-axis. 


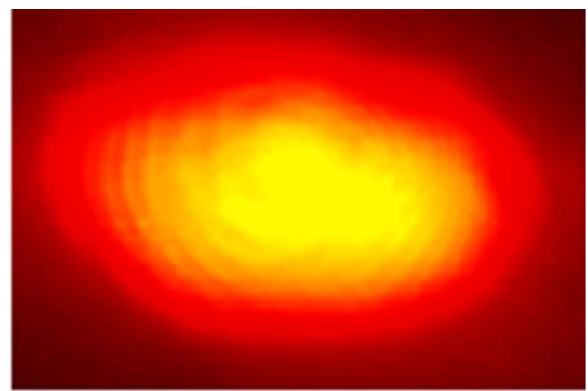

(a)

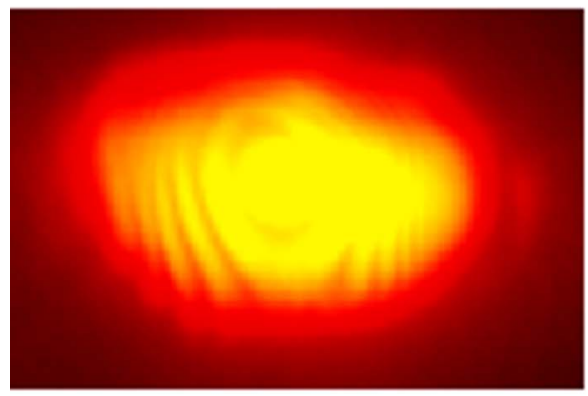

(b)

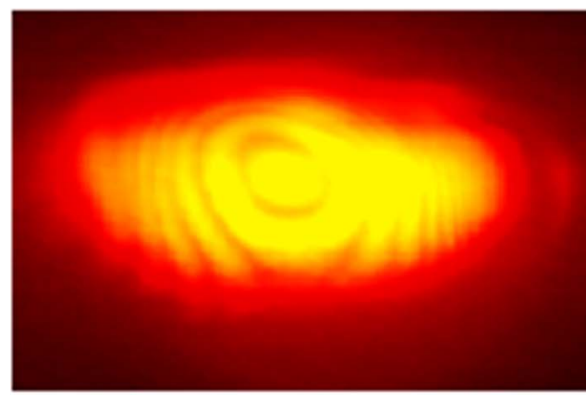

(c)

Fig. 4. (Color online) Interference fringes for linearly polarized light verifying the third and fourth Fresnel and Arago laws. (a) Polarizer $\mathrm{P}_{4}$ inserted and $\mathrm{P}_{1}$ removed, no recovery of fringes. (b) Introduction of $\mathrm{P}_{1}$ made interference fringes appear. (c) The sharpness increases when polarizers $P_{1}$ and $\mathrm{P}_{4}$ are present and producing light beams with the same direction of polarization

interference fringes were obtained with little sharpness [Fig. 4(a)]. This sharpness depended on the degree of correlation between the orthogonal components $\mathrm{E}_{\mathrm{x}}$ and $\mathrm{E}_{\mathrm{y}}$ of the electric field.

In the experimental arrangement for the verification of the third law (Fig. 3), a polarizer $\mathrm{P}_{1}$ with direction of polarization $45^{\circ}$ with respect to the $X$-axis was introduced before the beam splitter $\mathrm{BS}_{1}$. The fringes are shown in Fig. 4(b). When we rotated $\mathrm{P}_{1}$, the interference fringes appeared and disappeared on the observation plane R. Fringes with maximum sharpness were obtained [Fig. 4(c)] when $\mathrm{P}_{4}$ had the same direction of polarization as $\mathrm{P}_{1}\left(45^{\circ}\right.$ with respect to the $X$-axis). The orthogonal polarized components of light from $\mathrm{P}_{2}$ and $\mathrm{P}_{3}$, which were brought into same plane of polarization by $\mathrm{P}_{4}$, interfered and produced fringes because they were originally derived from the same polarized component of light (by polarizer $\mathrm{P}_{1}$ ). Thus the fourth law was also verified.

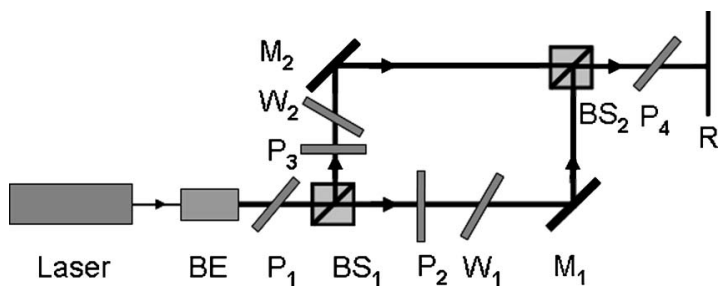

Fig. 5. Experimental arrangement of Mach-Zehnder interferometer for verifying the Fresnel-Arago laws using circular polarization. $W_{1}$ and $W_{2}$ are quarter wave plates with their optical axis $45^{\circ}$ with respect to the incident polarization of the light beam.

If we rotated polarizer $\mathrm{P}_{4}$, the fringes disappeared when the direction of polarization of $\mathrm{P}_{2}$ or $\mathrm{P}_{3}$ was orthogonal to $\mathrm{P}_{4}$. In these conditions one of the light beams was stopped by $\mathrm{P}_{4}$ due to cross-polarization.

The Fresnel and Arago laws were also verified for circularly polarized light. Two multiple order quarter wave plates (wavelength $=632.8 \mathrm{~nm}$ ) with optical axis $45^{\circ}$ (in the clockwise direction) with respect to the polarization of the incoming beam were introduced after polarizers $\mathrm{P}_{2}$ and $\mathrm{P}_{3}$ (see Fig. 5 ). The beams emerging after the quarter wave plates were right circularly polarized, and sharp interference fringes were produced on the observation plane [Fig. 6(a)]. Then one of the quarter wave plates $\mathrm{W}_{1}$ was rotated anticlockwise by $90^{\circ}$, producing a left circularly polarized light. The two beams with orthogonal circular polarization did not produce any fringes [Fig. 6(b)]. The fringes could be reproduced [Fig. 6(c)] if polarizer $\mathrm{P}_{4}$ (as in Fig. 3), with the same direction of polarization as $\mathrm{P}_{1}$, was introduced after beam-splitter $\mathrm{BS}_{2}$ as shown in Fig. 5. Removing $\mathrm{P}_{1}$ made the fringes vanish as shown in Fig. 6(d). These observations verify the four Fresnel-Arago laws for circularly polarized light.

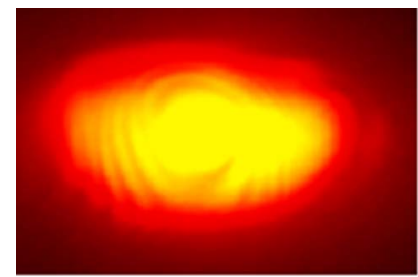

(a)

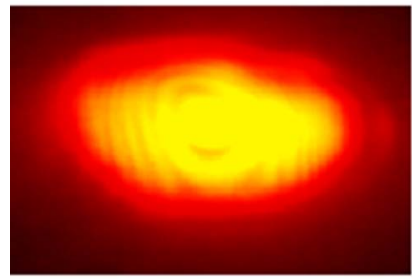

(c)

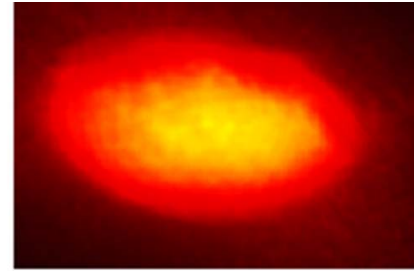

(b)

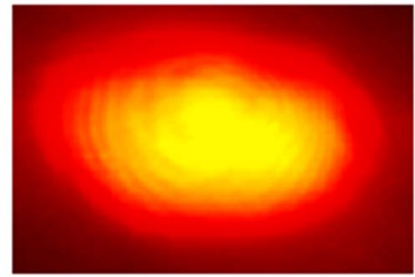

(d)
Fig. 6. (Color online) Interference fringes for circularly polarized light. (a) Fringes appear for both light beams with right circular polarization. (b) Fringes vanish if one of the two beams is made left circularly polarized. (c) Fringes reappear (introducing $\mathrm{P}_{1}$ and $\mathrm{P}_{4}$ ) when the light beams are brought into the same plane of polarization (originally derived from the same polarized component). (d) Removing $\mathrm{P}_{1}$ makes the fringes vanish. 


\section{ACKNOWLEDGMENTS}

The authors are thankful to the Director of the National Physical Laboratory, New Delhi for permission to publish this paper. Authors B.K. and N.S wish to thank CSIR for a Junior Research Fellowship.

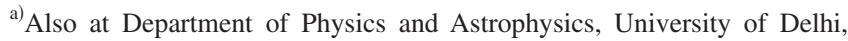
Delhi 110007, India.

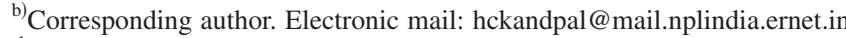

${ }^{1}$ D. F. J. Arago and A. J. Fresnel, "On the action of rays of polarized light upon each other," Ann. Chim. Phys. 2, 288-304 (1819).

${ }^{2}$ E. Whittaker, A History of the Theories of Aether and Electricity (Philosophical Society, New York, 1951), Vol. I.

${ }^{3}$ G. G. Stokes, "On the composition and resolution of streams of polarized light from different sources," Trans. Cambridge Philos. Soc. 9, 399-426 (1852).

${ }^{4} \mathrm{R}$. Hanau, "Interference of linearly polarized light with perpendicular polarizations," Am. J. Phys. 31, 303-304 (1963).

${ }^{5}$ E. Collet, "Mathematical formulation of the interference laws of Fresnel and Arago," Am. J. Phys. 39, 1483-1495 (1971).

${ }^{6}$ C. Brosseau, Fundamentals of Polarized Light: A Statistical Optics Approach (Wiley, New York, 1998)
${ }^{7}$ R. Barakat, "Analytic proof of the Arago Fresnel laws for the interference of polarized light," J. Opt. Soc. Am. A 10, 180-185 (1993).

${ }^{8}$ M. Mujat, A. Dogarin, and E. Wolf, "A law of interference of electromagnetic beams of any state of coherence and polarization and the Fresnel-Arago interference laws," J. Opt. Soc. Am. A 21, 2414-2417 (2004).

${ }^{9} \mathrm{E}$. Wolf, "Unified theory of coherence and polarization of random electromagnetic beams," Phys. Lett. A 312, 263-267 (2003).

${ }^{10} \mathrm{~W}$. R. Mellen, "Interference of linearly polarized light with perpendicular polarizations," Am. J. Phys. 30, 772 (1962).

${ }^{11}$ E. Fortin, "Direct demonstration of the Fresnel-Arago laws," Am. J. Phys. 38, 917-918 (1970).

${ }^{12} \mathrm{M}$. Henry, "Fresnel-Arago laws for interference in polarized light: A demonstration experiment," Am. J. Phys. 49, 690-691 (1981).

${ }^{13}$ J. L. Ferguson, "A simple, bright demonstration of the interference of polarized light," Am. J. Phys. 52, 1141-1142 (1984).

${ }^{14}$ M. Born and E. Wolf, Principles of Optics, 7th ed. (Cambridge U. P., Cambridge, 1999).

${ }^{15}$ L. Dettwiller, "Polarization state interference: A general investigation," Pure Appl. Opt. 6, 41-53 (1997).

${ }^{16} \mathrm{R}$. Castaneda, "Electromagnetic spatial coherence wavelets and the classical laws of polarization," Opt. Commun. 267, 4-13 (2006).

${ }^{17}$ P. Hariharan, Basics of Interferometry (Academic, San Diego, 1992).

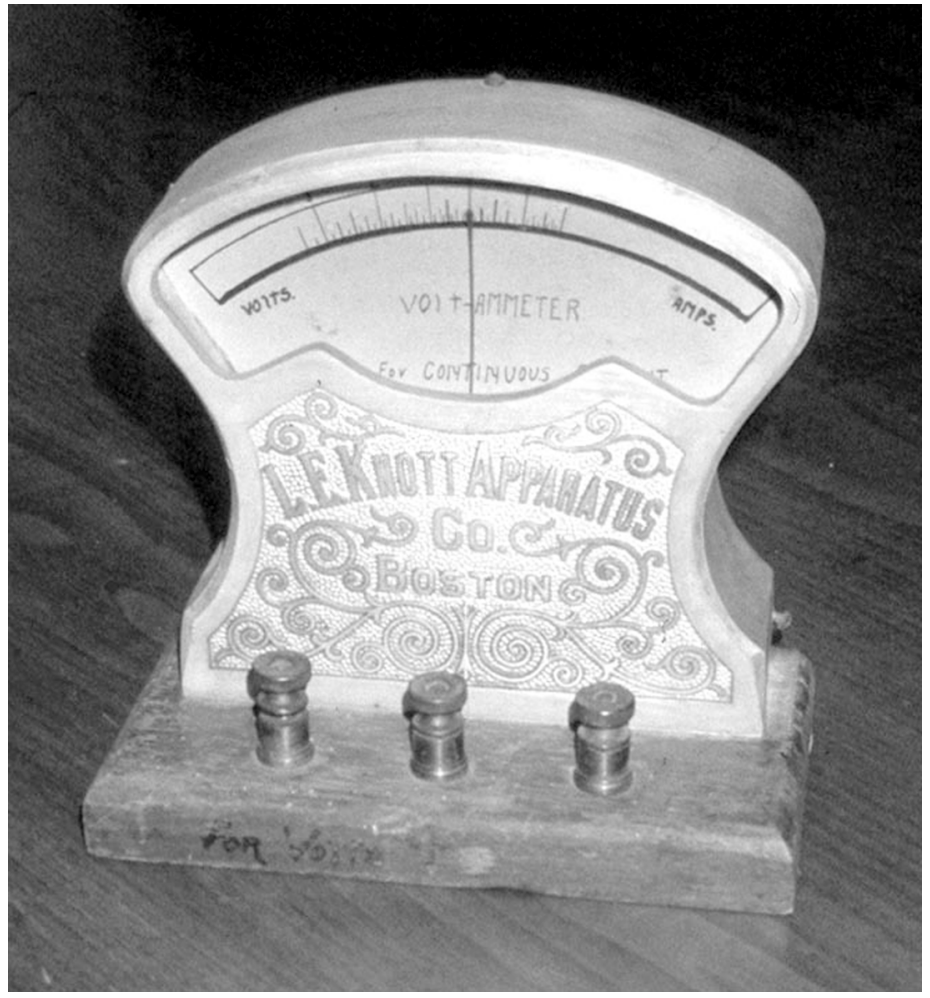

Upright Volt-Ammeter. This instrument has an unusual upright orientation. This example, at Fredonia University in western New York, has a hand-lettered scale, although one at Washington and Lee University has a printed scale. One of the front terminals is the low side for the meter movement. A second one has a high resistance connected to the movement through a high resistance multiplier for use as a voltmeter, and the third has a low resistance shunt that connected across the movement for use as an ammeter. The name of the maker, the E.L. Knott Apparatus Company of Boston is cast onto the aluminum case. (Photograph and Notes by Thomas B. Greenslade, Jr., Kenyon College) 